ESAIM: PROCEEDINGS AND SURVEYS, October 2015, Vol. 51, p. 1-23

A. Garivier et al, Editors

\title{
TRANSPORT INEQUALITIES AND CONCENTRATION OF MEASURE*
}

\author{
NATHAEL GOZLAN ${ }^{1}$
}

\begin{abstract}
We give a short introduction to the concentration of measure phenomenon and connect it with different functional inequalities (Poincaré, Talagrand and Log-Sobolev inequalities).

Résumé. Nous présentons une courte introduction au phénomène de concentration de la mesure et nous le mettons en lien avec diverses inégalités fonctionnelles (Inégalités de Poincaré, Talagrand et Log-Sobolev).
\end{abstract}

\section{INTRODUCTION}

This paper provides a short survey of some aspects of the concentration of measure phenomenon. Concentration of measure inequalities appeared in the seventies as a key tool in the study of asymptotic geometry of Banach spaces with the alternative seminal proof by Milman [90] of a celebrated result by Dvoretzky [43] on the existence of high dimensional nearly Euclidean sections of convex bodies. The subject was then deeply investigated during the last decades (see for instance [91], [105], [62, Chapter 3.5], [77]) and is still a very active research area both from a theoretical and an applied point of view.

The paper is organized as follows. In the first section, we recall the classical Sudakov-Tsirelson/Borell isoperimetric result in Gauss space. Then we give general definitions and examples about concentration of measure and we recall some classical applications of this phenomenon in probability and analysis. In the second part, we illustrate the interplay between functional inequalities and the concentration of measure phenomenon. In particular, we present two results from [53] and [61] characterizing respectively Gaussian and exponential type dimension-free concentration in terms of transport inequalities.

\section{The CONCEntration of MEASURe Phenomenon}

This section is devoted to classical aspects of the concentration of measure phenomenon. First, we recall the example of Gaussian measures. Then we give general definitions about concentration inequalities and illustrate them by various classical examples. We end the section by recalling three classical applications of concentration of measure in analysis and probability theory.

\subsection{The example of Gaussian measure}

In all the paper, we will denote by $\gamma^{d}$ the standard $d$-dimensional Gaussian measure on $\mathbb{R}^{d}$. The space $\mathbb{R}^{d}$ will always be equipped with its standard Euclidean norm denoted by $|\cdot|$.

\footnotetext{
* Supported by the grants ANR 2011 BS01 007 01, ANR 10 LABX-58

1 Université Paris Est Marne la Vallée - Laboratoire d'Analyse et de Mathématiques Appliquées (UMR CNRS 8050), 5 bd Descartes, 77454 Marne la Vallée Cedex 2, France. e-mail: natael.gozlan@u-pem.fr
}

(c) EDP Sciences, SMAI 2015 
If $A \subset \mathbb{R}^{d}$ is a Borel set, the boundary measure (also called Minkowski content) of $A$ is defined by

$$
\gamma_{+}^{d}(\partial A)=\liminf _{r \rightarrow 0^{+}} \frac{\gamma^{d}\left(A_{r}\right)-\gamma^{d}(A)}{r},
$$

where $A_{r}$ is the $r$-enlargement of $A$ defined as follows

$$
A_{r}=\left\{x \in \mathbb{R}^{d}: \exists y \in A,|x-y| \leq r\right\}
$$

The Gaussian isoperimetric problem: For a given Gaussian volume, what are the sets having minimal boundary measure?

As is well known, for the usual Lebesgue measure on $\mathbb{R}^{d}$ isoperimetric sets are balls. In Gauss space, the isoperimetric sets are half-spaces, $i$.e. sets of the form $H=\left\{x \in \mathbb{R}^{d}: x \cdot u \leq r\right\}$, where $u \in \mathbb{R}^{d}$ and $r \in \mathbb{R}$.

Theorem 1.1 (Sudakov and Tsirel'son [103], Borell [29]). Half-spaces are solutions of the Gaussian isoperimetric problem: If $A \subset \mathbb{R}^{d}$ is a Borel set and $H$ a half-space such that $\gamma^{d}(H)=\gamma^{d}(A)$, then

$$
\gamma_{+}^{d}(\partial A) \geq \gamma_{+}^{d}(\partial H)
$$

This remarkable result admits different proofs. The original proof of $[29,103]$ deduced the Gaussian isoperimetric result from Levy's isoperimetric theorem on the sphere $\mathbb{S}^{n-1} \subset \mathbb{R}^{n}$ equipped with its uniform probability measure $\sigma_{n-1}$, according to which spherical caps are isoperimetric sets. The link between uniform measures on spheres and Gaussian measures is given by the following elementary observation going back at least to Poincaré: letting, for $n \geq d, \pi_{n, d}: \mathbb{R}^{n} \rightarrow \mathbb{R}^{d}:\left(x_{1}, \ldots, x_{n}\right) \mapsto \sqrt{n}\left(x_{1}, \ldots, x_{d}\right)$, then

$$
\pi_{n, d \#} \sigma_{n-1} \rightarrow \gamma^{d}, \quad \text { weakly as } n \rightarrow \infty,
$$

where, in all the paper, $T_{\#} \mu$ denotes the push-forward of a probability measure $\mu$ under a map $T$.

Other proofs have been developed by Ehrard [44,45] (based on a symmetrization argument in Gauss space), Bakry and Ledoux [7] (based on a semigroup interpolation technique) or Bobkov [16] (based on an inequality on the two points space and a tensorization argument coupled with the central limit theorem - see also [13]). It was also shown by Carlen and Kerce [31] that half-spaces are the only isoperimetric sets. Very recently, the stability of the Gaussian isoperimetric problem was considered in [35, 47, 92].

Now let us express the isoperimetric inequality (1) in a more quantitative form. First, it is clear by rotational invariance, that one can always assume that $H$ is of the form $H=\left\{x \in \mathbb{R}^{d}: x_{1} \leq r\right\}$. For such $H$, it holds $\gamma^{d}(H)=\Phi(r)$, with

It follows that

$$
\Phi(r)=\frac{1}{\sqrt{2 \pi}} \int_{-\infty}^{r} e^{-t^{2} / 2} d t, \quad r \in \mathbb{R} .
$$

$$
\gamma_{+}^{d}(\partial H)=\Phi^{\prime}(r)=\frac{1}{\sqrt{2 \pi}} e^{-r^{2} / 2}, \quad r \in \mathbb{R} .
$$

Therefore, Gaussian isoperimetry can be restated as follows.

Corollary 1.2. For any Borel set $A \subset \mathbb{R}^{d}$,

$$
\gamma_{+}^{d}(\partial A) \geq \Phi^{\prime} \circ \Phi^{-1}\left(\gamma^{d}(A)\right)
$$

Furthermore, observing that at least formally $\gamma_{+}^{d}\left(\partial A_{r}\right)=\frac{d}{d r} \gamma^{d}\left(A_{r}\right)$ (details can be found in [22] or [77]), one obtains the following integrated version of Gaussian isoperimetry:

Corollary 1.3. For any Borel set $A \subset \mathbb{R}^{d}$,

$$
\gamma^{d}\left(A_{r}\right) \geq \Phi\left(r+\Phi^{-1}\left(\gamma^{d}(A)\right)\right), \quad \forall r \geq 0
$$


Let us finally specialize to the situation where $\gamma^{d}(A) \geq 1 / 2$, then $\Phi^{-1}\left(\gamma^{d}(A)\right) \geq 0$ and we end up with the so-called Gaussian concentration inequality

Corollary 1.4 (Gaussian concentration inequality). For any Borel set $A \subset \mathbb{R}^{d}$ such that $\gamma^{d}(A) \geq 1 / 2$,

$$
\gamma^{d}\left(A_{r}\right) \geq \Phi(r), \quad \forall r \geq 0 .
$$

In practice, one usually bounds $\Phi$ from below as follows: $\Phi(r) \geq 1-\frac{1}{2} e^{-r^{2} / 2}, r \geq 0$. This yields to the following explicit (but slightly weaker) form of (3):

$$
\gamma^{d}\left(A_{r}\right) \geq 1-\frac{1}{2} e^{-r^{2} / 2}, \quad \forall r \geq 0, \quad \forall A \subset \mathbb{R}^{d} \text { with } \gamma^{d}(A) \geq 1 / 2 .
$$

The remarkable feature of Inequalities (3) and (4) is that the dimension of the space does not appear in the inequality. This independence with respect to the dimension has been successfully exploited in numerous applications in analysis, probability or statistics in large dimension.

\subsection{Concentration property for general probability measures}

Let us now recall the classical definitions about concentration properties of probability measures in a general setting. In what follows, $(\mathcal{X}, d)$ will be a Polish space, $\mu$ a probability measure on $\mathcal{X}$ and $\alpha: \mathbb{R}^{+} \rightarrow[0,1]$ a non-increasing function.

Definition 1.5 (Concentration of measure).

- One says that $\mu$ satisfies the concentration of measure property with the concentration profile $\alpha$ if for all $A \subset \mathcal{X}$ with $\mu(A) \geq 1 / 2$, it holds

$$
\mu\left(A_{r}\right) \geq 1-\alpha(r), \quad r \geq 0,
$$

where

$$
A_{r}=\{x \in \mathcal{X}: d(x, A) \leq r\}, \quad r \geq 0 .
$$

- One says that $\mu$ satisfies the dimension-free concentration of measure property with the concentration profile $\alpha$, if for all $n \in \mathbb{N}^{*}$, the product measure $\mu^{\otimes n}$ satisfies the concentration of measure property with the profile $\alpha$ on $\mathcal{X}^{n}$ equipped with the distance

$$
d_{2}(x, y)=\left[\sum_{i=1}^{n} d^{2}\left(x_{i}, y_{i}\right)\right]^{1 / 2}, \quad x, y \in \mathcal{X}^{n} .
$$

Let us illustrate this definition by recalling the concentration properties of classical probability measures (see [77] for a proof).

\section{Example 1.6.}

(1) The standard Gaussian measure $\gamma$ on $\mathbb{R}$ satisfies the dimension-free concentration property with the concentration profile

$$
\alpha(r)=\bar{\Phi}(r)=1-\Phi(r)=\frac{1}{\sqrt{2 \pi}} \int_{r}^{+\infty} e^{-u^{2} / 2} d u \leq \frac{1}{2} e^{-r^{2} / 2}, \quad r \geq 0 .
$$

(2) More generally, if $\mu(d x)=e^{-V(x)} d x$ is a probability measure on a complete, connected, Riemannian manifold $\mathcal{X}$ equipped with its geodesic distance which satisfies the Bakry-Emery $\operatorname{CD}(K, \infty)$ condition

$$
\text { Hess } V+\operatorname{Ric} \geq K \text { Id, }
$$


for some $K>0$, where Ric denotes the Ricci tensor, then $\mu$ satisfies the concentration property with a profile of the form

for some $r_{o}>0$.

$$
\alpha(r)=e^{-K \frac{\left[r-r_{o}\right]_{+}^{2}}{2}}, \quad r \geq 0
$$

(3) For $p \in[1,2)$, the probability measure $\mu_{p}$ defined on $\mathbb{R}$ by

$$
\mu_{p}(d x)=\frac{1}{Z_{p}} e^{-|x|^{p} / p} d x .
$$

satisfies the dimension-free concentration property with a profile of the form

$$
\alpha_{p}(r)=e^{-a_{p}\left[r-r_{p}\right]_{+}^{p}}, \quad r \geq 0
$$

for some $r_{p}>0$.

Concentration of measure can be alternatively defined in terms of deviation inequalities for Lipschitz functions (see e.g. [77, Proposition 1.3] for a proof).

Proposition 1.7. The following propositions are equivalent

(1) The probability measure $\mu$ satisfies the concentration property with the profile $\alpha$.

(2) For all 1-Lipschitz function $f$ on $\mathcal{X}$,

$$
\mu\left(f>m_{f}+r\right) \leq \alpha(r), \quad \forall r \geq 0,
$$

where $m_{f}$ denotes a median of $f$ with respect to $\mu$.

In particular, if $\mu$ satisfies the dimension-free concentration property with the profile $\alpha$, then, for all $n \in \mathbb{N}^{*}$

$$
\mu^{n}\left(\left|f-m_{f}\right|>r\right) \leq 2 \alpha(r), \quad r \geq 0
$$

for all $f: \mathcal{X}^{n} \rightarrow \mathbb{R}$ which is 1-Lipschitz with respect to the $d_{2}$ distance (5).

This type of inequalities (which can be interpreted as the fact that Lipschitz functions are essentially constant when the underlying probability measure is strongly concentrated) is commonly used in applications, as we shall see in the next section.

Remark 1.8. Sometimes, it is more convenient to consider deviation inequalities with respect to the mean of $f$ instead of its median. If the concentration profile $\alpha$ is integrable on $[0, \infty)$ (which is in general the case), one can do the following: integrating (6), and using an integration by parts, yields to

$$
\int\left|f-m_{f}\right| d \mu^{n}=\int_{0}^{+\infty} \mu^{n}\left(\left|f-m_{f}\right|>r\right) d r \leq 2 \int_{0}^{+\infty} \alpha(r) d r:=r_{o} .
$$

So assuming $r_{o}<\infty$, we get $\left|\int f d \mu-m_{f}\right| \leq r_{o}$ and inserting this inequality into (6), yields to

$$
\mu^{n}\left(\left|f-\int f d \mu\right|>r\right) \leq 2 \alpha\left(r-r_{o}\right), \quad r \geq r_{o}
$$

We refer to [77, Propositions 1.7 and 1.8] for further results in the same spirit.

\subsection{Classical applications}

In this section, we recall three classical examples of applications of concentration ideas. 


\subsubsection{Dvoretzky's theorem}

Let us begin by recalling the proof by V. Milman [90] of the following celebrated result of Dvoretzky.

Theorem 1.9 (Dvoretzky [43]). Let $\|\cdot\|$ be a norm on $\mathbb{R}^{d}$. For all $\varepsilon>0$, there exists a $k$-dimensional subspace $H=\operatorname{Vect}\left\{v_{1}, \ldots, v_{k}\right\}$ with $k=\lfloor\eta(\varepsilon) \log (d)\rfloor$ and $\eta(\varepsilon)=a \frac{\varepsilon^{2}}{\log (1 / \varepsilon)}$ for some universal constant a $>0$, such that

$$
(1-\varepsilon)|t| \leq\left\|\sum_{i=1}^{k} t_{i} v_{i}\right\| \leq(1+\varepsilon)|t|, \quad \forall t \in \mathbb{R}^{k} .
$$

In other words, if $B=\left\{x \in \mathbb{R}^{d}:\|x\| \leq 1\right\}$ denotes the unit ball of $\left(\mathbb{R}^{d},\|\cdot\|\right)$ and $\mathcal{E}$ the following ellipsoid $\mathcal{E}=\left\{\sum_{i=1}^{k} t_{i} v_{i}:|t| \leq 1\right\} \subset H$, then the section $B \cap H$ is nearly spherical in the sense that

$$
\frac{1}{1+\varepsilon} \mathcal{E} \subset B \cap H \subset \frac{1}{1-\varepsilon} \mathcal{E}
$$

Sketch of proof. Milman's original proof used concentration of measure on the sphere. Here, we follow the proof by Pisier [95] involving concentration for Gaussian random vectors. To simplify, we assume that $\|\cdot\|=\|\cdot\|_{\infty}$, and we refer to $[77,95]$ for a complete proof. One has to show that there exist $v_{1}, \ldots, v_{k}$ linearly independent such that

$$
(1-\varepsilon) \leq\left\|\sum_{i=1}^{k} t_{i} v_{i}\right\|_{\infty} \leq(1+\varepsilon), \quad t \in \mathbb{S}^{k-1} .
$$

For a fixed $t \in \mathbb{S}^{k-1}$, the map $F_{t}\left(x_{1}, \ldots, x_{k}\right)=\left\|\sum_{i=1}^{k} t_{i} x_{i}\right\|_{\infty}$ is 1-Lipschitz with respect to the Euclidian norm on $\left(\mathbb{R}^{d}\right)^{k}$. Therefore, the Gaussian concentration property (4) implies (in the form given by $(7)$ ) that if $X_{i}$ is a sequence of i.i.d random variables with law $\gamma^{d}$,

$$
\mathbb{P}\left(\left|F_{t}\left(X_{1}, \ldots, X_{k}\right)-m\right|>r\right) \leq 2 e^{-\left(r-r_{o}\right)^{2} / 2}, \quad \forall r \geq r_{o},
$$

where $m=\mathbb{E}\left[F_{t}\left(X_{1}, \ldots, X_{k}\right)\right]$ and $r_{o}=2 \int_{0}^{+\infty} e^{-r^{2} / 2} d r=\sqrt{2 \pi}$. Since $-\frac{1}{2}\left(r-r_{o}\right)^{2} \leq \frac{1}{2} r_{o}^{2}-\frac{r^{2}}{4}$, for $r \geq r_{o}$, we get

$$
\mathbb{P}\left(\left|F_{t}\left(X_{1}, \ldots, X_{k}\right)-m\right|>r\right) \leq 2 e^{\frac{1}{2} r_{o}^{2}} e^{-\frac{r^{2}}{4}},
$$

for all $r \geq r_{o}$, and the inequality is still true for $r \leq r_{o}$. So, letting $C=2 e^{\frac{1}{2} r_{o}^{2}}$, it holds

$$
\mathbb{P}\left(\left|F_{t}\left(X_{1}, \ldots, X_{k}\right)-m\right|>\varepsilon m\right) \leq C e^{-\varepsilon^{2} m^{2} / 4}, \quad \forall \varepsilon>0 .
$$

One can show that $m \simeq \sqrt{\log d}$ (see e.g. [95, Lemma 4.4]). Now, if $N \subset \mathbb{S}^{k-1}$ is a finite subset, the union bound immediately gives

$$
\mathbb{P}\left(\sup _{t \in N}\left|F_{t}\left(X_{1}, \ldots, X_{k}\right)-m\right|>\varepsilon m\right) \leq C|N| e^{-c \varepsilon^{2} \log d},
$$

where $c>0$ is some universal numeric constant. We conclude from this, that if the cardinal $|N|$ of $N$ is such that $C|N| e^{-c \varepsilon^{2} \log d}<1$, the vectors $v_{i}=X_{i} / m$ satisfy

$$
(1-\varepsilon) \leq\left\|\sum_{i=1}^{k} t_{i} v_{i}\right\|_{\infty} \leq(1+\varepsilon), \quad \forall t \in N
$$

with a positive probability. Let us take for $N$ an $\varepsilon$-net of $\mathbb{S}^{k-1}$. A classical argument shows that $N$ can be chosen of cardinal $|N| \leq\left(1+\frac{2}{\varepsilon}\right)^{k}$ (see e.g. [95, Lemma 4.10]) and one sees that taking $k=\eta(\varepsilon) \log d$, with $\eta(\varepsilon)$ as in the statement, one has $C|N| e^{-c \varepsilon^{2} \log d}<1$. Then the proof consists in extending (8) to arbitrary $t \in S^{k-1}$, with $\varepsilon$ replaced by $3 \varepsilon$ (see e.g. [95, Lemma 4.11]). 


\subsubsection{Deviations inequalities for the empirical measure of large random matrices}

Let $M^{n}=\frac{1}{\sqrt{n}}\left[M_{i, j}\right]_{i, j}$ be a sequence of symmetric $n \times n$ random matrices where $\left\{M_{i, j} ; i<j \in \mathbb{N}\right\}$ and $\left\{M_{i, i} ; i \in \mathbb{N}\right\}$ are two independent families of i.i.d random variables such that $\mathbb{E}\left[M_{1,1}\right]=\mathbb{E}\left[M_{1,2}\right]=0$ and $\mathbb{E}\left[M_{1,2}^{2}\right]=1$ and $\mathbb{E}\left[M_{1,1}^{2}\right]<\infty$. Denote by $\lambda_{1} \leq \ldots \leq \lambda_{n} \in \mathbb{R}$ the (random) spectrum of $M^{n}$. According to the famous Wigner's theorem (see e.g. [2]), with probability one, the empirical measure $L_{n}$ defined by

$$
L_{n}=\frac{1}{n} \sum_{i=1}^{n} \delta_{\lambda_{i}}
$$

converges weakly to the semicircular law $d \sigma(x)=\frac{1}{2 \pi} \sqrt{4-x^{2}} \mathbf{1}_{[-2,2]}(x) d x$, when $n \rightarrow \infty$. In other words, for any bounded continuous function $f$ on $\mathbb{R}$,

$$
\int f d L_{n}=\frac{1}{n} \sum_{i=1}^{n} f\left(\lambda_{i}\right) \rightarrow \int f d \sigma, \quad \text { a.s when } n \rightarrow+\infty
$$

Denote by $\mu_{1}$ the common law of the $M_{i, i}$ 's and by $\mu_{2}$ the common law of the $M_{i, j}$ 's, $i<j$.

Theorem 1.10 (Guionnet-Zeitouni [66]). Let $f: \mathbb{R} \rightarrow \mathbb{R}$ be a 1-Lipschitz function and $F_{n}=\left\langle f, L_{n}\right\rangle=$ $\frac{1}{n} \sum_{i=1}^{n} f\left(\lambda_{i}\right)$. If $\mu_{1} \otimes \widetilde{\mu_{2}}$, where $\widetilde{\mu_{2}}$ is the pushforward of $\mu_{2}$ under the map $x \mapsto \sqrt{2} x$ satisfies the dimensionfree concentration property with a profile of the form $\alpha(r)=e^{-\frac{1}{C}\left[r-r_{o}\right]^{2}}$, then

$$
\mathbb{P}\left(\left|F_{n}-m_{n}\right|>r\right) \leq 2 e^{-\frac{1}{C} n^{2}\left[r-r_{o}\right]_{+}^{2}}, \quad \forall n \geq 1, \quad \forall r \geq 0,
$$

where $m_{n}$ is the median of $F_{n}$.

\section{Remark 1.11.}

- The median $m_{n}$ can be replaced by the expectation $\mathbb{E}\left[F_{n}\right]$. Moreover, under mild assumptions on $f$, it is possible to give explicit bounds for $\left|\mathbb{E}\left[F_{n}\right]-\int f d \sigma\right|$ (see [66] and the references therein for details).

- It will follow from Theorems 2.7 and 2.5 that assuming that $\mu_{1} \otimes \widetilde{\mu_{2}}$ satisfies the dimension-free concentration of measure property with the profile $\alpha(r)=e^{-\frac{1}{C}\left[r-r_{o}\right]^{2}}$ is the same as assuming the same property separately for $\mu_{1}$ and for $\widetilde{\mu_{2}}$.

Proof. For any symmetric matrix $A$ with real coefficients, let us define $f_{n}(A)=\frac{1}{n} \sum_{i=1}^{n} f\left(\lambda_{i}(A)\right)$, where $\lambda_{1}(A) \leq$ $\ldots \leq \lambda_{n}(A)$ is the spectrum of $A$. For all symmetric matrices $M, M^{\prime}$, it holds

$$
\left|F_{n}(M / \sqrt{n})-F_{n}\left(M^{\prime} / \sqrt{n}\right)\right| \leq \frac{1}{n} \sum_{i=1}^{n}\left|\lambda_{i}(M / \sqrt{n})-\lambda_{i}\left(M^{\prime} / \sqrt{n}\right)\right| \leq \frac{1}{\sqrt{n}}\left(\sum_{i=1}^{n}\left|\lambda_{i}(M / \sqrt{n})-\lambda_{i}\left(M^{\prime} / \sqrt{n}\right)\right|^{2}\right)^{1 / 2} .
$$

According to a classical inequality by Hoffmann-Wielandt (see e.g. [2, Lemma 2.1.19]), it turns out that

$$
\sum_{i=1}^{n}\left|\lambda_{i}(A)-\lambda_{i}(B)\right|^{2} \leq \operatorname{Tr}\left((A-B)^{2}\right)=\sum_{i=1}^{n}\left(A_{i i}-B_{i i}\right)^{2}+2 \sum_{i<j}\left(A_{i j}-B_{i j}\right)^{2} .
$$

Therefore,

$$
\left|F_{n}(M / \sqrt{n})-F_{n}\left(M^{\prime} / \sqrt{n}\right)\right| \leq \frac{1}{n}\left(\sum_{i=1}^{n}\left(M_{i i}-M_{i i}^{\prime}\right)^{2}+2 \sum_{i<j}\left(M_{i j}-M_{i j}^{\prime}\right)^{2}\right)^{1 / 2}
$$


Therefore, if $E=\left\{M: F_{n}(M / \sqrt{n}) \leq m_{n}\right\}$, then taking $M^{\prime} \in A$ and optimizing yields

$$
F_{n}(M / \sqrt{n}) \leq m_{n}+\frac{1}{n} d(\widetilde{M}, \widetilde{E}),
$$

where $\widetilde{M}=\left(\sqrt{2-\delta_{i j}} M_{i, j}\right)_{i \leq j} \in \mathbb{R}^{n(n+1) / 2}, \widetilde{E}=\{\widetilde{M}: M \in E\}$ and $d(\widetilde{M}, \widetilde{E})=\inf _{M^{\prime} \in A}\left|\widetilde{M}-\widetilde{M^{\prime}}\right|$, where $|\cdot|$ denotes the $\ell_{2}$-norm on the space of matrices. Using the concentration property satisfied by the law of $\widetilde{M}$, it follows that

$$
\mathbb{P}\left(F_{n}\left(M^{n}\right)>m_{n}+r\right) \leq \mathbb{P}(d(\widetilde{M}, \widetilde{E})>n r) \leq e^{-\frac{1}{C} n^{2}\left[r-r_{o}\right]_{+}^{2}} .
$$

A similar reasoning shows that the same inequality holds for deviations under the median, which concludes the proof.

The power $n^{2}$ appearing in Theorem 1.10 is in accordance with the large-deviation estimates obtained by Ben-Arous and Guionnet [14] in the case of Gaussian entries (and other models of random matrices). More precisely, if $\mu_{1}=\mathcal{N}(0,1)$ and $\mu_{2}=\mathcal{N}(0,2)$, the sequence of random matrices $M^{n}$ is the so called Gaussian Orthogonal Ensemble and the following large deviation principle holds : For any set $A \subset \mathcal{P}(\mathbb{R})$ (the space of all Borel probability measures on $\mathbb{R}$ ), it holds

$$
-\inf _{\nu \in \operatorname{int}(A)} I(\nu) \leq \liminf _{n \rightarrow \infty} \frac{1}{n^{2}} \log \mathbb{P}\left(L_{n} \in A\right) \leq \limsup _{n \rightarrow \infty} \frac{1}{n^{2}} \log \mathbb{P}\left(L_{n} \in A\right) \leq-\inf _{\nu \in \operatorname{cl}(A)} I(\nu),
$$

where closure $\mathrm{cl}$ and interior int are defined with respect to the usual weak convergence topology of $\mathcal{P}(\mathbb{R})$ and the rate function $I: \mathcal{P}(\mathbb{R}) \rightarrow[0, \infty]$ is defined by

$$
I(\nu)=\frac{1}{4} \int x^{2} d \nu(x)-\frac{1}{2} \iint \log (|x-y|) d \nu(x) d \nu(y)-\frac{3}{8} .
$$

One can show that $I(\nu) \geq 0$ with equality if and only if $\nu$ is the semicircular law $\sigma$. In particular if $A$ is a set of the form $\left\{\nu \in \mathcal{P}(\mathbb{R}):\left|\int f d \nu-\int f d \sigma\right|>r\right\}, r>0$, for some bounded Lipschitz function $f$, then $\mathbb{P}\left(L_{n} \in A\right) \simeq e^{-a n^{2}}$, for some $a>0$. This shows the optimality of the bound in Theorem 1.10. See also the end of Section 2.1.2 for another application of these (large) deviations inequalities.

\subsubsection{The central limit theorem for log-concave random vectors}

A probability measure $\mu$ on $\mathbb{R}^{d}$ is said log-concave, if it has a density of the form $e^{-V}$ with a convex function $V: \mathbb{R}^{d} \rightarrow \mathbb{R} \cup\{+\infty\}$. It is isotropic if $\int x \mu(d x)=0$ and $\int x_{i} x_{j} \mu(d x)=\delta_{i j}$.

The following striking result due to Klartag [70] (see also [49]) uses a concentration idea going back to Sudakov [102] to show that when $X$ is isotropic and log-concave then, for most of the directions $\theta \in \mathbb{S}^{d-1}$ the law of $X \cdot \theta$ is approximately Gaussian. We refer to the surveys by Klartag [72] and Guédon [64] on the subject.

Theorem 1.12 (Klartag [70]). Let $X$ be a random vector with an isotropic log-concave law. There exists a subset $\Theta$ of $\mathbb{S}^{d-1}$ with $\sigma_{d-1}(\Theta) \geq 1-e^{-c \sqrt{d}}$ and such that for all $\theta \in \Theta$,

$$
\sup _{t \in \mathbb{R}}|\mathbb{P}(X \cdot \theta \leq t)-\Phi(t)| \leq \frac{C}{d^{\alpha}},
$$

where $\Phi(t)=\frac{1}{\sqrt{2 \pi}} \int_{-\infty}^{t} e^{-u^{2} / 2} d u$ and where $c, C, \alpha>0$ are universal numerical constants.

Sketch of the proof. (1) The first step (the most difficult one) consists in showing that

$$
\operatorname{Var}\left(|X|^{2}\right) \leq C d^{2-\alpha}
$$


where $C, \alpha>0$ are universal numeric constants. As a consequence, noting that by isotropicity $\mathbb{E}\left[|X|^{2}\right]=d$ and applying Markov's inequality together with the elementary inequality $\left.|| X\right|^{2}-d|\geq \sqrt{d}||X|-\sqrt{d} \mid$, one sees easily that the vector $X$ satisfies the following deviation inequality

$$
\mathbb{P}(|| X|-\sqrt{d}|>t \sqrt{d}) \leq \frac{C}{t^{2} d^{\alpha}}, \quad \forall t>0 .
$$

It follows in particular that $X$ is concentrated in a thin shell around the origin: with high probability $|X| \simeq$ $\sqrt{d}+o(\sqrt{d})$. The validity of (10) for all isotropic log-concave vectors was first conjectured in [4] and then proved by Klartag in [70].

(2) The next step is a general argument using the concentration of measure on the sphere. It first appeared in a paper by Sudakov [102] and was then developed in various contexts in $[4,18,19,41,109]$. Let us take a 1-Lipschitz test function $f: \mathbb{R} \rightarrow \mathbb{R}$ and set $F(\theta)=\mathbb{E}[f(X \cdot \theta)]$ and explain why $F(\theta) \simeq \int f d \gamma$ for most of the $\theta$ 's. It is not difficult to check that the function $F$ is 1 -Lipschitz on $\mathbb{S}^{d-1}$, so, according to the concentration result on the sphere, it is essentially equal to its mean (with respect to $\sigma_{d-1}$ ). Therefore, for most of the directions $\theta$, it holds

$$
\begin{aligned}
F(\theta) & \simeq \int F(\omega) \sigma_{d-1}(d \omega) \quad(\text { concentration on the sphere) } \\
& =\mathbb{E}\left[\int f(X \cdot \omega) \sigma_{d-1}(d \omega)\right] \quad \text { (Fubini) } \\
& =\mathbb{E}\left[\int f\left(|X| \omega_{1}\right) \sigma_{d-1}(d \omega)\right] \quad \text { (rotational invariance) } \\
& \left.\simeq \int f\left(\sqrt{d} \omega_{1}\right) \sigma_{d-1}(d \omega) \quad \text { (concentration for }|X|\right) .
\end{aligned}
$$

Finally, it follows from Poincaré observation (2) that

$$
\int f\left(\sqrt{d} \omega_{1}\right) \sigma_{d-1}(d \omega) \simeq \frac{1}{\sqrt{2 \pi}} \int f(x) e^{-x^{2} / 2} d x .
$$

Remark 1.13. The currently best exponent $\alpha$ in the inequality (10) is $\alpha=1 / 3$ and was obtained by Guédon and Milman [65]. It is conjectured that $\alpha=1$ (variance conjecture).

\section{FunCtional APPROACHES OF ThE CONCENTRATION OF MEASURE PHENOMENON}

In recent years, various methods were developed to prove concentration of measure inequalities. Let us mention, in particular:

- Use of geometric inequalities (Brunn-Minkowski and Prekopa-Leindler inequalities) [25, 85],

- Use of functional inequalities (Poincaré, Log-Sobolev, transport inequalities ... ) $[63,76,106]$,

- Induction methods [105],

- Martingale inequalities [86,87],

- Entropic method [83,84],

- Coupling methods [34,68],

- ...

We refer to the books by Ledoux [77] and by Boucheron, Lugosi and Massart [6] and the references therein for a panoramic view on the subject and for more information on these particular directions.

In what follows, we will focus on the approach based on functional inequalities. As we shall see in the case of Exponential and Gaussian dimension-free concentration, functional inequalities do not only provide a method 
to get dimension-free concentration inequalities but are actually an equivalent form of this phenomenon. In this description of the dimension-free concentration of measure phenomenon the class of transport inequalities introduced by Marton [80-82] and Talagrand [106] will play a central role. We refer the interested reader to [56], [8], [27], [108] and [6] for surveys of some of the recent developments in the field.

\subsection{Talagrand's transport inequalities and Gaussian dimension-free concentration}

In this section, we introduce Talagrand's transport inequality $\mathbf{T}_{2}$ and recall classical arguments by Marton and Talagrand showing that $\mathbf{T}_{2}$ implies dimension-free Gaussian concentration. Then we sketch the proof of a result by the author [53] showing that the converse implication also holds. We conclude by recalling different necessary and sufficient conditions for Talagrand's inequality.

\subsubsection{Definitions and historical references}

Talagrand's inequality compares two very natural functionals defined on the space of probability measures: the Monge-Kantorovich quadratic transport cost and the relative entropy (a.k.a Kullbak-Leibler divergence) that we shall now define.

Definition 2.1. Let $\mu, \nu$ be two probability measures on $\mathcal{X}$.

- The quadratic transport cost between $\mu$ and $\nu$ is defined by

$$
\mathcal{T}_{2}(\mu, \nu):=\inf _{\pi \in P(\mu, \nu)} \iint_{\mathcal{X} \times \mathcal{X}} d(x, y)^{2} \pi(d x d y),
$$

where $P(\mu, \nu)=\left\{\pi \in \mathcal{P}(\mathcal{X} \times \mathcal{X})\right.$ such that $\pi_{1}=\mu$ and $\left.\pi_{2}=\nu\right\}$ is the set of all couplings between $\mu$ and $\nu$. The so called Wasserstein distance is defined by $W_{2}(\nu, \mu)=\sqrt{\mathcal{T}_{2}(\nu, \mu)}($ see $[96,107,108])$.

- The relative entropy of $\nu$ with respect to $\mu$ is defined by

$$
\mathrm{H}(\nu \mid \mu)=\int \log \left(\frac{d \nu}{d \mu}\right) d \nu, \text { if } \nu \ll \mu, \text { and }+\infty, \text { otherwise. }
$$

Definition 2.2 (Talagrand's transport inequality). A probability measure $\mu$ on $\mathcal{X}$ satisfies Talagrand's inequality with the constant $C>0$ (in short $\mathbf{T}_{2}(C)$ ), if

$$
W_{2}(\mu, \nu) \leq \sqrt{C \mathrm{H}(\nu \mid \mu)}, \quad \forall \nu \in \mathcal{P}(\mathcal{X}) .
$$

The ancestor of all transport inequalities is the following very useful inequality due to Csiszar [38], Kullback [74], Pinsker [94] comparing total variation distance to relative entropy:

$$
\|\mu-\nu\|_{\text {T.V. }}^{2} \leq \frac{1}{2} \mathrm{H}(\nu \mid \mu),
$$

where we recall that total variation distance between $\mu$ and $\nu$ is defined by $\|\mu-\nu\|_{\text {T.V. }}=\sup _{A \subset \mathcal{X}}|\mu(A)-\nu(A)|$. Equivalently,

$$
\|\mu-\nu\|_{\text {T.V. }}=\inf _{(X, Y)} \mathbb{P}(X \neq Y),
$$

where the infimum runs over the set of couples of random variables $(X, Y)$ with $X$ distributed according to $\mu$ and $Y$ according to $\nu$ (see e.g. [84, Lemma 2.20]). This last formula shows that the total variation distance corresponds to an optimal transport cost with respect to the Hamming distance cost function $d(x, y)=1_{x \neq y}$.

Marton was the first to make a connection between this type of inequalities and the concentration of measure phenomenon. In her first works on the subject [80-82], she studied refined versions of (11) involving weighted forms of the Hamming distance (and in particular, their tensorization properties) and recovered from them some of the deep concentration of measures inequalities by Talagrand [105] (the so called convex distance inequalities). 
This was then amplified by Dembo [39] with in particular multi-marginal refinements of (11). See also the papers by Samson [98-100] for different extensions of Marton's inequalities (in particular in a dependent setting). Some weighted versions of the inequality (11) can be found in $[28,52]$.

The first transport inequality involving the Euclidean distance (in place of the Hamming distance) is due to Talagrand.

Theorem 2.3 (Talagrand [106]). The standard Gaussian measure $\gamma^{d}$ on $\left(\mathbb{R}^{d},|\cdot|\right)$ satisfies $\mathbf{T}_{2}(2)$.

This result was then extended to probability measures with a density of the form $e^{-V}$ with a uniformly convex potential $V$ by [93], [25] or [36]. Let us mention that Maurey proved in [85] the so called $(\tau)$-property for the Gaussian measure (and other log-concave measures). It was realized in [21] (see also [56, Section 8.1]) that the $(\tau)$-property is an equivalent dual version of the transport inequality. Talagrand's inequality admits many variants involving different cost functions. We refer to [56] for a description of some of these results. Let us mention two very natural extensions. Talagrand's inequality was extended to abstract Wiener spaces by Gentil [50], Feyel and Ustünel [48] and Djellout, Guillin and Wu [42]). It was also introduced in the framework of free probability by Biane and Voiculescu [15] (see also Theorem 2.9 below).

\subsection{2. $\mathbf{T}_{2}$ and the dimension-free Gaussian concentration property}

Let us recall the nice and simple argument due to Marton that extracts concentration properties from transport inequalities. Here, we restrict for simplicity to the case of the inequality $\mathbf{T}_{2}$ but the argument is general (see e.g. [56]).

Theorem 2.4. If $\mu$ satisfies $\mathbf{T}_{2}(C)$, then $\mu$ satisfies the concentration property with the profile

$$
\alpha(r)=e^{-\frac{1}{C}\left[r-r_{o}\right]_{+}^{2}}, \quad r \geq 0,
$$

with $r_{o}=\sqrt{C \log (2)}$.

Proof. Let us take $A \subset \mathcal{X}$ such that $\mu(A) \geq 1 / 2$ and set $B=\mathcal{X} \backslash A^{r}, r>0$ and consider $d \mu_{A}=\frac{\mathbf{1}_{A}}{\mu(A)} d \mu$ and $d \mu_{B}=\frac{\mathbf{1}_{B}}{\mu(B)} d \mu$. Then it holds

$$
W_{2}\left(\mu_{A}, \mu_{B}\right) \leq W_{2}\left(\mu_{A}, \mu\right)+W_{2}\left(\mu_{B}, \mu\right) \leq \sqrt{C \mathrm{H}\left(\mu_{A} \mid \mu\right)}+\sqrt{C \mathrm{H}\left(\mu_{B} \mid \mu\right)} \leq r_{o}+\sqrt{-C \log (\mu(B))} .
$$

It follows easily from the definition that $W_{2}\left(\mu_{A}, \mu_{B}\right) \geq d(A, B) \geq r$. Therefore

$$
\mu(B) \leq \exp \left(-\frac{1}{C}\left(r-r_{o}\right)^{2}\right), \quad \forall r \geq r_{o}
$$

It turns out that dimension-free concentration can be derived from $\mathbf{T}_{2}$. This follows from the following tensorization property discovered by Marton and Talagrand.

Theorem 2.5. If $\mu$ satisfies $\mathbf{T}_{2}(C)$, then for all $n \in \mathbb{N}^{*}, \mu^{\otimes n}$ satisfies $\mathbf{T}_{2}(C)$ on $\left(\mathcal{X}^{n}, d_{2}\right)$.

Proof. It is enough to prove the $n=2$ case. If $\nu \in \mathcal{P}(\mathcal{X} \times \mathcal{X})$, one writes its disintegration with respect to its first variable as follows

$$
\nu\left(d x_{1} d x_{2}\right)=\nu\left(d x_{2} \mid x_{1}\right) \nu_{1}\left(d x_{1}\right) .
$$

The proof then relies on the following disintegration formulas for $\mathrm{H}$ :

$$
\mathrm{H}(\nu \mid \mu \otimes \mu)=\mathrm{H}\left(\nu_{1} \mid \mu\right)+\int \mathrm{H}\left(\nu\left(\cdot \mid x_{1}\right) \mid \mu\right) \nu_{1}\left(d x_{1}\right) .
$$


For the transport cost $\mathcal{T}_{2}$ the same holds with an inequality $\leq$. Therefore, if $\mu$ satisfies $\mathbf{T}_{2}(C)$, we get

$$
\begin{aligned}
\mathcal{T}_{2}(\nu, \mu \otimes \mu) & \leq \mathcal{T}_{2}\left(\nu_{1}, \mu\right)+\int \mathcal{T}_{2}\left(\nu\left(\cdot \mid x_{1}\right), \mu\right) \nu_{1}\left(d x_{1}\right) \\
& \leq C\left(\mathrm{H}\left(\nu_{1} \mid \mu\right)+\int \mathrm{H}\left(\nu\left(\cdot \mid x_{1}\right) \mid \mu\right) \nu_{1}\left(d x_{1}\right)\right) \\
& =C \mathrm{H}(\nu \mid \mu \otimes \mu),
\end{aligned}
$$

and so $\mu^{2}$ satisfies $\mathbf{T}_{2}(C)$.

In particular, if $\mu$ satisfies $\mathbf{T}_{2}$, then applying Theorem 2.4 to $\mu^{n}$ for all $n \geq 1$, one obtains the following

Corollary 2.6. If $\mu$ satisfies $\mathbf{T}_{2}(C)$, then $\mu$ satisfies the dimension-free concentration property with the profile

$$
\alpha(r)=e^{-\frac{1}{C}\left[r-r_{o}\right]_{+}^{2}}, \quad r \geq 0 .
$$

A remarkable feature of the inequality $\mathbf{T}_{2}$ is that the converse implication also holds.

Theorem 2.7 (Gozlan [53]). If $\mu$ satisfies the dimension-free Gaussian concentration property with a profile of the form

$$
\alpha(r)=\exp \left(-\frac{1}{C}\left[r-r_{o}\right]_{+}^{2}\right), \quad r \geq 0
$$

then $\mu$ satisfies $\mathbf{T}_{2}(C)$.

This result thus shows that the inequality $\mathbf{T}_{2}$ is the minimal functional description of the dimension-free concentration of measure phenomenon. Note in particular that the best constant in $\mathbf{T}_{2}$ is always equal to the best constant in the exponential profile (12). See [57] for another proof of Theorem 2.7.

Sketch of proof. The idea is to estimate from above and from below the following probability of rare event

$$
\mathbb{P}\left(W_{2}\left(L_{n}, \mu\right)>t\right), \quad t \geq 0
$$

where $L_{n}=\frac{1}{n} \sum_{i=1}^{n} \delta_{X_{i}}$ and $X_{i}$ is an i.i.d sequence of law $\mu$.

(1) The first estimate (from above) is given by Gaussian concentration: roughly speaking, when $n$ is large, it holds

$$
\mathbb{P}\left(W_{2}\left(L_{n}, \mu\right)>t\right) \leq e^{-n \frac{t^{2}}{C}} .
$$

(this follows easily from the fact that the random variable $W_{2}\left(L_{n}, \mu\right)$ is a $1 / \sqrt{n}$ Lipschitz function of the $\left.X_{i}^{\prime} s\right)$

(2) The second estimate is given by Sanov large deviation theorem (see e.g. [40, Theorem 6.2.10]):

$$
-\inf \left\{\mathrm{H}(\nu \mid \mu): W_{2}(\nu, \mu)>t\right\} \leq \liminf _{n \rightarrow+\infty} \frac{1}{n} \log \mathbb{P}\left(W_{2}\left(L_{n}, \mu\right)>t\right)
$$

The comparison of these two informations immediately gives Talagrand's inequality

$$
W_{2}^{2}(\nu, \mu) \leq C \mathrm{H}(\nu \mid \mu), \quad \forall \nu \in \mathcal{P}(\mathcal{X})
$$

Remark 2.8. One could ask if the choice of the $W_{2}$ distance is really important in the definition of Talagrand's inequality. Let us for instance consider the $W_{1}$ version of Talagrand's inequality (the so called $\mathbf{T}_{1}$ inequality): a probability $\mu$ satisfies $\mathbf{T}_{1}(C)$ if

$$
W_{1}(\nu, \mu) \leq \sqrt{C \mathrm{H}(\nu \mid \mu)}, \quad \forall \nu \in \mathcal{P}(\mathcal{X})
$$


The $\mathbf{T}_{1}$ inequality is also connected to Gaussian concentration (Marton's argument applies exactly as before) but not to dimension-free concentration (since the tensorization property of $\mathbf{T}_{1}$ depends on the dimension). See $[42,56]$ for details. It turns out that $\mathbf{T}_{1}$ is much weaker than $\mathbf{T}_{2}$. Indeed, it was first proved in [42] that $\mu$ satisfies $\mathbf{T}_{1}(C)$ if and only if there is some $a>0$ such that $\int e^{a d^{2}\left(x_{o}, x\right)} \mu(d x)<\infty$ (for some and thus all $x_{o} \in \mathcal{X}$ ). Note that for instance a probability supported in (say) two disjoint closed balls in $\mathbb{R}^{d}$ will satisfy the integrability condition, but not the Poincaré inequality and thus not $\mathbf{T}_{2}$ (see Theorem 2.10 below).

The proof above applies in other contexts. It was recently used by Riedel [97] to derive Talagrand inequality in an abstract Wiener space from Borell's concentration inequality (thus recovering the result from [48]). It can also be used to give a new proof of the following result by Biane and Voiculescu (this was suggested to us by Michel Ledoux some years ago).

Theorem 2.9 (Biane and Voiculescu [15]). The semi-circular law $d \sigma(x)=\frac{1}{2 \pi} \sqrt{4-x^{2}} \mathbf{1}_{[-2,2]}(x) d x$ satisfies the following transport inequality

$$
W_{2}(\nu, \sigma)^{2} \leq 4 I(\nu), \quad \forall \nu \in \mathcal{P}(\mathbb{R})
$$

where $I(\nu)=\frac{1}{4} \int x^{2} d \nu-\frac{1}{2} \iint \log (|x-y|) d \nu(x) d \nu(y)-3 / 8$.

Proof. We use the notations introduced in Section 1.3.2. As in the proof of Theorem 2.7, the idea is to bound from above and from below the probability of the event $\left\{W_{2}\left(L_{n}, \sigma\right)>t\right\}$, where $L_{n}=\frac{1}{n} \sum_{i=1}^{n} \delta_{\lambda_{i}}$ and $\lambda_{1} \leq \ldots \leq \lambda_{n}$ are the eigenvalues of $M^{n}$.

(1) To bound the probability from above, we use concentration. First observe that

$$
\left|W_{2}\left(L_{n}(\omega), \sigma\right)-W_{2}\left(L_{n}\left(\omega^{\prime}\right), \sigma\right)\right| \leq W_{2}\left(L_{n}(\omega), L_{n}\left(\omega^{\prime}\right)\right) \leq \frac{1}{\sqrt{n}} \sqrt{\sum_{i=1}^{n}\left|\lambda_{i}(\omega)-\lambda_{i}\left(\omega^{\prime}\right)\right|^{2}} .
$$

So reasoning as in the proof of Theorem 1.10, we see that

$$
\mathbb{P}\left(W_{2}\left(L_{n}, \sigma\right)>m_{n}+t\right) \leq e^{-\frac{n^{2}}{4} t^{2}}, \quad \forall t \geq 0 .
$$

where $m_{n}$ denotes the median of $W_{2}\left(L_{n}, \sigma\right)$ (here $\mu_{1}=\widetilde{\mu_{2}}=\mathcal{N}(0,2)$ ). It is not difficult to see that $m_{n} \rightarrow 0$ when $n \rightarrow \infty$.

(2) On the other hand, the bound from below is provided by the large deviation principle (9) :

$$
\liminf _{n \rightarrow \infty} \frac{1}{n^{2}} \log \mathbb{P}\left(W_{2}\left(L_{n}, \sigma\right)>t\right) \geq-\inf \left\{I(\nu): W_{2}(\nu, \sigma)>t\right\} .
$$

(observe that the set $W_{2}(\cdot, \sigma)>t$ is open).

Comparing the two bounds above, easily yields to

$$
\frac{1}{4} W_{2}(\nu, \sigma)^{2} \leq I(\nu),
$$

for all probability $\nu$ on $\mathbb{R}$, which concludes the proof.

The proof above is close but different from the proof by Hiai, Petz and Ueda [67] also involving large deviations. See also [78] for a direct transport proof of Biane-Voiculescu transport inequality, and [79] for a transport inequality involving the $W_{1}$ distance. 


\subsubsection{Necessary and sufficient conditions for $\mathbf{T}_{2}$}

In this section, we recall the position of $\mathbf{T}_{2}$ in the hierarchy of classical Sobolev type functional inequalities and we give some specific criteria ensuring that a given probability measure satisfies the inequality $\mathbf{T}_{2}$.

First let us recall a celebrated result of Otto and Villani which compares Talagrand's inequality $\mathbf{T}_{2}$ to two other well known functional inequalities: Poincaré inequality and Log-Sobolev inequality. We recall that a probability measure $\mu$ on $\mathbb{R}^{d}$ (or more generally on a Riemannian manifold) satisfies the Poincaré inequality with the constant $C>0$ (in short $\mathbf{P I}(C)$ ) if

$$
\operatorname{Var}_{\mu}(f) \leq \frac{C}{2} \int|\nabla f|^{2} d \mu, \quad \forall \text { smooth } f
$$

and that it satisfies the Log-Sobolev inequality (in short $\mathbf{L S I}(C)$ ) if

$$
\operatorname{Ent}_{\mu}\left(f^{2}\right) \leq C \int|\nabla f|^{2} d \mu, \quad \forall \text { smooth } f
$$

where, for all non-negative function $g, \operatorname{Ent}_{\mu}(g)=\int g \log \left(\frac{g}{\int g d \mu}\right) d \mu$.

We refer to [3] and [6] for an introduction to these (and to many other) inequalities and their applications in terms of convergence of semigroups toward equilibrium, hypercontractivity, etc... The natural hierarchy between LSI and $\mathbf{P I}$ is the following: $\mathbf{L S I}(C) \Rightarrow \mathbf{P I}(C / 2)$. The following result shows that $\mathbf{T}_{2}$ is in-between $\mathbf{P I}$ and LSI.

Theorem 2.10 (Otto-Villani [93]). Let $\mu$ be an absolutely continuous probability measure on a complete connected Riemannian manifold

(1) If $\mu$ satisfies the logarithmic Sobolev inequality $\mathbf{L S I}(C)$ then $\mu$ satisfies $\mathbf{T}_{2}(C)$.

(2) If $\mu$ satisfies $\mathbf{T}_{2}(C)$ then $\mu$ satisfies Poincaré inequality $\mathbf{P I}(C / 2)$.

In particular, if $\mu(d x)=e^{-V(x)} d x$ satisfies the Bakry-Emery $\operatorname{CD}(K, \infty)$ condition [5]

$$
\operatorname{Ric}+\operatorname{Hess} V \geq K
$$

with $K>0$, then it satisfies $\mathbf{L S I}(1 / K)$ and so $\mathbf{T}_{2}(1 / K)$. Direct transport proofs (on $\mathbb{R}^{d}$ ) of these results can be found in [36].

Remark 2.11. Note that since LSI implies $\mathbf{T}_{2}$, it also implies, according to Corollary 2.6, dimension-free Gaussian concentration of measure. Actually, this conclusion also follows from a classical elementary argument due to Herbst [77, Theorem 5.3] which is also recalled in Section 2.3.1. It is also shown in [93] that for logconcave measures $\mathbf{T}_{2}$ gives back LSI (see [93, Corollary 3.1] for a precise statement valid more generally for probability measures with a density of the form $e^{-V}$ with Hess $V$ bounded from below). Though very close from each other, the inequalities LSI and $\mathbf{T}_{2}$ are not equivalent. This was proved by Cattiaux and Guillin [32] who exhibited the first example of a probability measure satisfying $\mathbf{T}_{2}$ but not $\mathbf{L S I}$. Other examples can be found in [55] and [12]. To conclude about the exact position of $\mathbf{T}_{2}$ with respect to LSI, let us finally mention that $\mathbf{T}_{2}$ is in fact equivalent to a restricted version of LSI. We refer to the series of papers by the author in collaboration with Roberto and Samson [58-60].

Now let us present some specific necessary or sufficient conditions for $\mathbf{T}_{2}$.

In dimension 1 a necessary and sufficient condition was obtained by the author in [55]. We will denote by $\mu_{1}$ the symmetric exponential probability measure

$$
\mu_{1}(d x)=\frac{1}{2} e^{-|x|} d x
$$


We will also denote by $F_{\mu}$ the cumulative distribution function of a probability measure $\mu$ on $\mathbb{R}$, defined by $\left.\left.F_{\mu}(x)=\mu(]-\infty, x\right]\right), x \in \mathbb{R}$ and by $F_{\mu}^{-1}$ its generalized inverse defined by $F_{\mu}^{-1}(t)=\inf \left\{x \in \mathbb{R}: F_{\mu}(x) \leq t\right\}$, $t \in(0,1)$.

Theorem 2.12 (Gozlan [55]). Let $\mu$ be a probability measure on $\mathbb{R}$. The following are equivalent

(1) There exists $C>0$ such that $\mu$ satisfies $\mathbf{T}_{2}(C)$,

(2) There exist $D, a>0$ such that $\mu$ satisfies Poincaré inequality with the constant $D$ and the map

$$
T=F_{\mu}^{-1} \circ F_{\mu_{1}}
$$

which is the unique left-continuous non-decreasing map transporting $\mu_{1}$ on $\mu$, satisfies the following condition

$$
|T(x)-T(y)| \leq a(\sqrt{|x-y|}+1), \quad \forall x, y \in \mathbb{R} .
$$

Moreover, there exists a universal numeric constant $\kappa>1$ such that

$$
\frac{1}{\kappa} \max \left(D_{\mathrm{opt}}, a_{\mathrm{opt}}^{2}\right) \leq C_{\mathrm{opt}} \leq \kappa \max \left(D_{\mathrm{opt}}, a_{\mathrm{opt}}^{2}\right)
$$

where $D_{\mathrm{opt}}$ and $a_{\mathrm{opt}}$ denotes respectively the best constants in Poincaré inequality and in (15).

One can deduce from the preceding result an explicit sufficient condition on the density of $\mu$ which was first obtained by Cattiaux and Guillin in [32].

Corollary 2.13 (Cattiaux and Guillin [32]). Let $\mu$ be a probability measure on $\mathbb{R}$ of the form $\mu(d x)=e^{-V(x)} d x$, with $V$ an even function of class $\mathcal{C}^{2}$ and such that $V^{\prime \prime} /\left(V^{\prime}\right)^{2}(x) \rightarrow 0$ as $x \rightarrow+\infty$. If $V$ is such that

$$
V^{\prime}(x) \geq \lambda x, \quad \forall x \geq x_{o} \geq 0,
$$

for some $\lambda>0$ and $x_{o} \geq 0$, then $\mu$ satisfies $\mathbf{T}_{2}(C)$ for some $C>0$.

This last result was then generalized in arbitrary dimension by Cattiaux, Guillin and Wu [33].

Theorem 2.14 (Cattiaux, Guillin and $\mathrm{Wu}[33]$ ). Let $\mu$ be a probability measure on $\mathbb{R}^{d}$ of the form $\mu(d x)=$ $e^{-V(x)} d x$, with $V$ of class $\mathcal{C}^{2}$. If $V$ is such that

$$
(1-\varepsilon)|\nabla V|^{2}-\Delta V \geq \lambda|x|^{2}, \quad \forall|x| \geq R
$$

for some $\lambda>0, \varepsilon \in] 0,1\left[\right.$ and $R>0$, then $\mu$ satisfies $\mathbf{T}_{2}(C)$ for some $C>0$.

Note that for other transport-entropy inequalities on $\mathbb{R}^{d}$, similar sufficient conditions can be found in [54].

\subsection{Poincaré inequality and dimension free concentration}

In this section, we present the links between Poincaré inequality (13) and dimension-free concentration. The main result of the section shows that Poincaré inequality is a necessary and sufficient condition for this phenomenon to hold. Then we recall a result by Bobkov, Gentil and Ledoux showing that Poincaré inequality can be represented as a transport inequality. We also make a comparison between our main result and a result by E. Milman establishing the equivalence between concentration and Poincaré inequality for Log-concave probability measures. 


\subsubsection{From Poincaré inequality to exponential dimension-free concentration}

The following result shows that Poincaré inequality implies exponential dimension-free concentration. The first result in this direction goes back to the works of Gromov and Milman [63] and Borovkov and Utev [30]. See also subsequent developments by Aida and Stroock [1], Bobkov and Ledoux [24] and Schmuckenschläger [101]. Note that in the paper by Bobkov and Ledoux, a sharper concentration result à la Talagrand (involving enlargements of sets with a mixing of $\ell_{1}$ and $\ell_{2}$ balls) is deduced from Poincaré.

Theorem 2.15 (Gromov and Milman [63], Borovkov and Utev [30]). Let $\mu$ be a probability measure on $\mathbb{R}^{d}$. If $\mu$ satisfies $\mathbf{P I}(C)$, then $\mu$ satisfies the dimension-free concentration of measure property with an exponential profile of the form

$$
\alpha(r)=\exp \left(-\frac{a}{\sqrt{C}}\left[r-r_{o}\right]_{+}\right)
$$

where $r_{o}=\sqrt{C} b$ and $a, b$ are universal numeric constants.

We refer to [77, Corollary 3.2] for the elementary proof of this result.

\subsubsection{Characterization of the dimension-free concentration of measure phenomenon}

The following result obtained in [61] establishes a strong converse to the preceding result. It shows that the class of all probability measures satisfying a non-trivial dimension free concentration of measure property is equal to the class of probability measures satisfying Poincaré inequality.

Theorem 2.16 (Gozlan, Roberto and Samson [61]). Let $\mu$ be a probability measure on $\mathbb{R}^{d}$; if $\mu$ satisfies a dimension-free concentration of measure inequality with a profile $\alpha$ such that $\alpha(r)<1 / 2$ for at least one value of $r>0$, then $\mu$ satisfies $\mathbf{P I}(C)$ with the constant

$$
C=\left(\inf \left\{\frac{r}{\bar{\Phi}^{-1}(\alpha(r))}: r>0 \text { s.t. } \alpha(r)<1 / 2\right\}\right)^{2},
$$

where

$$
\bar{\Phi}(r)=\frac{1}{\sqrt{2 \pi}} \int_{r}^{+\infty} e^{-u^{2} / 2} d u .
$$

In the same spirit, let us mention that dimension-free concentration with respect to $\ell_{\infty}$ - enlargements (instead of $\ell_{2}$ as in the preceding result) has been characterized for 1-dimensional probability measures by Bobkov and Houdré [23]. For 1-dimensional measures again, Bobkov and Götze [21] showed that dimension-free concentration restricted to convex sets and complements of convex sets was equivalent to Poincaré inequality restricted to convex functions.

The proof of Theorem 2.16 relies on a combination of the central limit theorem (giving rise to the function $\bar{\Phi})$ and Hamilton-Jacobi equation (introducing the $|\nabla f|^{2}$ in the inequality). Let us mention that Theorem 2.16 is stated in $\mathbb{R}^{d}$ only for convenience, but its conclusion is actually true on any metric space (with a proper definition of Poincaré inequality). See [61] for details.

\section{Remark 2.17.}

(1) If $\mu=\gamma$ is the standard Gaussian measure on $\mathbb{R}$, then according to Corollary 1.4 it satisfies the dimension-free concentration of measure property with the profile $\alpha=\bar{\Phi}$. Thus inserting this $\alpha$ in (16), one recovers that $\gamma$ satisfies Poincaré with the constant $C=1$ which is the optimal one.

(2) The preceding result applies even in the case of an elementary profile $\alpha \equiv 1 / 2$ on $\left[0, r_{o}\right.$ [ and $\alpha \equiv \lambda_{o} \in$ $\left[0,1 / 2\left[\right.\right.$ on $\left[r_{o}, \infty[\right.$.

An immediate corollary of the above results is the following characterization of dimension-free exponential concentration. 
Corollary 2.18. A probability measure $\mu$ satisfies Poincaré inequality if and only if it satisfies the dimension free concentration property with an exponential profile.

If $\mu$ is a probability measure on $\mathbb{R}^{d}$, denote by $\alpha_{\mu}$ its asymptotic concentration profile defined by

$$
\alpha_{\mu}(r)=\sup _{n \in \mathbb{N}^{*}} \sup _{A \subset\left(\mathbb{R}^{d}\right)^{n}: \mu^{n}(A) \geq 1 / 2} 1-\mu^{n}\left(A_{r}\right), \quad r \geq 0
$$

In other words, the function $\alpha_{\mu}$ is the best function $\alpha$ such that $\mu$ satisfies the dimension-free concentration of measure phenomenon. Note that this function can be the constant $1 / 2$ (in which case $\mu$ does not enjoy any non-trivial dimension-free concentration result). The following result shows that in case $\alpha_{\mu}$ is not trivial, then it is in between exponential and Gaussian profiles.

Theorem 2.19. Let $\mu$ be a probability measure on $\mathbb{R}^{d}$.

(1) Either $\alpha_{\mu} \equiv 1 / 2$, or there exist $a>0, r_{o} \geq 0$ such that $\alpha_{\mu}(r) \leq e^{-a\left[r-r_{o}\right]_{+}}$.

(2) Assume $d=1$ and $\mu$ has expectation $m$ and variance $\sigma^{2}$. Either $\mu=\mathcal{N}\left(m, \sigma^{2}\right)$ or $\alpha_{\mu}(r)>\bar{\Phi}(r / \sigma)$, for all $r>0$.

In particular, the first point means that dimension-free concentration is always at least exponential. This was already observed by Talagrand in [104, Proposition 5.1].

Proof. Point (1) is a direct consequence of Theorems 2.16 and 2.15. Let us prove Point (2). According to a well know result by Borovkov and Utev [30] (see e.g. [26] for a proof), at fixed variance the Gaussian probability measure possesses the least Poincaré constant. Therefore, if $\mu$ is not Gaussian, the constant $C$ of (16) is greater than $\sigma^{2}$ which implies that $\alpha_{\mu}(r)>\bar{\Phi}(r / \sigma)$, for all $r>0$.

\subsubsection{A formal comparison with a result by E. Milman}

Theorem 2.16 is strongly reminiscent of the following result by E. Milman [89] which proves that any nontrivial concentration result for a log-concave probability measure gives an estimate of the Poincaré constant of the measure.

Theorem 2.20 (E. Milman [88,89]). Let $\mu$ be a log-concave probability measure on $\mathbb{R}^{d}$. If $\mu$ satisfies the following concentration inequality: there are some $r_{o}>0$ and $\lambda_{o} \in[0,1 / 2)$ such that

$$
\mu\left(A_{r_{o}}\right) \geq 1-\lambda_{o}, \quad \forall A \subset \mathbb{R}^{d} \text {, s.t. } \mu(A) \geq 1 / 2,
$$

then $\mu$ satisfies $\mathbf{P I}(C)$ with $C=4\left(\frac{r_{o}}{1-2 \lambda_{o}}\right)^{2}$.

The papers $[88,89]$ contain a nice general equivalence between concentration of measure properties, functional inequalities and isoperimetric type inequalities under curvature conditions. For example, it follows from this general result that for a log-concave probability measure, Gaussian concentration implies Log-Sobolev inequality (with a quantitative and dimension free link between the constants). See also [57] for a different proof based on transport inequalities. A similar result was already obtained by Wang [110] (see also [11, 17]) but under the assumption of a finite exponential moment of order 2 (assumption which has the disadvantage to yield to a dimension dependent constant in LSI).

\subsubsection{Poincaré inequality expressed as a transport inequality.}

The result below is due to Bobkov, Gentil and Ledoux [20]. It shows that Poincaré inequality is equivalent to some transport inequality involving a cost function which is quadratic for small displacements of mass and linear for large displacements. 
Theorem 2.21 (Bobkov, Gentil and Ledoux [20]). A probability measure $\mu$ on $\mathbb{R}^{d}$ satisfies $\mathbf{P I}(C)$ for some constant $C>0$ if and only if there exists some $D>0$ such that $\mu$ satisfies the following transport inequality

$$
\mathcal{T}(\nu, \mu) \leq \mathrm{H}(\nu \mid \mu), \quad \forall \nu \in \mathcal{P}\left(\mathbb{R}^{d}\right),
$$

where

$$
\mathcal{T}(\nu, \mu)=\inf \mathbb{E}\left[\min \left(D|X-Y| ; D^{2}|X-Y|^{2}\right)\right],
$$

where the infimum runs over the set of all couples of random variables $(X, Y)$, with $X$ distributed according to $\nu$ and $Y$ according to $\mu$.

Note that the link between the constants $C$ and $D$ is explicit and quantitative (see [20, Corollary 5.1] for a precise version).

Summarizing the contents of Corollary 2.6, Theorem 2.7 and Corollary 2.18, we see that exponential and Gaussian dimension-free concentration are both equivalent to a certain transport inequality.

Identifying a functional inequality that would be equivalent to dimension-free concentration with a profile of the form

$$
\alpha_{p}(r)=e^{-a\left[r-r_{o}\right]_{+}^{p}}, \quad r \geq 0
$$

with $p \in] 1,2[$, is still an open problem. Several functional inequalities give concentration rates between exponential and Gaussian (see in particular $[9,51,75]$ ). Nevertheless, considering the two extreme cases $p=1$ and $p=2$, a natural conjecture is that this concentration phenomenon is equivalent to a transport inequality with a cost function of the form

$$
c_{p}(x, y)=\min \left(|x-y|^{2} ;|x-y|^{p}\right), \quad x, y \in \mathbb{R}^{d} .
$$

\subsection{Applications}

To conclude this survey, we show two consequences of Theorems 2.7 and 2.16.

\subsubsection{A proof of Otto-Villani Theorem using concentration}

The theorem by Otto and Villani 2.10 showing the implication LSI $\Rightarrow \mathbf{T}_{2}$ admits at least three different proofs. The original proof by Otto and Villani [93] was based on calculations of the derivatives of the relative entropy and the transport cost along an interpolation given by the solutions of Fokker-Planck equation. Then Bobkov, Gentil and Ledoux [20] proposed another proof based on a dual formulation of the inequality $\mathbf{T}_{2}$ and Hamilton-Jacobi equations. A third proof was given by the author [53] relying on the characterization of $\mathbf{T}_{2}$ in terms of dimension free concentration. This is the one we sketch below.

Proof of Theorem 2.10. (LSI $\Rightarrow \mathbf{T}_{2}$ ) If $\mu$ satisfies $\mathbf{L S I}(C)$, then, according to a well known elementary argument due to Herbst (see e.g. [77, Theorem 5.1] or below), it satisfies a dimension-free concentration inequality with the profile

$$
\alpha(r)=e^{-\frac{1}{C}\left[r-r_{o}\right]_{+}^{2}}, \quad r \geq 0 .
$$

According to the characterization of $\mathbf{T}_{2}$ given in Theorem 2.7, $\mu$ satisfies $\mathbf{T}_{2}(C)$.

$\left(\mathbf{T}_{2} \Rightarrow \mathbf{P I}\right)$ If $\mu$ satisfies $\mathbf{T}_{2}$, then according to Corollary 2.6, it satisfies a dimension-free concentration inequality with a Gaussian profile of the form

$$
\alpha(r)=e^{-\frac{1}{C}\left[r-r_{o}\right]_{+}^{2}}, \quad r \geq 0 .
$$

According to the characterization of $\mathbf{P I}, \mu$ satisfies Poincaré inequality with the constant $D$ such that

$$
\sqrt{D} \leq \inf \left\{\frac{r}{\bar{\Phi}^{-1}(\alpha(r))}: r>0 \text { t.q } \alpha(r)<1 / 2\right\} \leq \frac{1}{\sqrt{2}} \lim _{r \rightarrow \infty} \frac{r}{\sqrt{-\log \alpha(r)}}=\sqrt{C / 2} .
$$


For the sake of completeness, we sketch the proof of Herbst's argument.

Proposition 2.22. If $\mu$ satisfies $\mathbf{L S I}(C)$, then it satisfies the dimension-free concentration inequality with the profile $\alpha(r)=e^{-\frac{1}{C}\left[r-r_{o}\right]_{+}^{2}}$, with $r_{o}=\sqrt{2 \log (C)}$.

Proof. Let $g$ be a bounded, 1-Lipschitz function on $\mathbb{R}^{d}$ and define for all $\lambda>0, Z(\lambda)=\int e^{\lambda g} d \mu$. Applying the log-Sobolev inequality (14) to $f=e^{\lambda g / 2}$, we get

$$
\lambda \int g e^{\lambda g} d \mu-\log \left(\int e^{\lambda g} d \mu\right) \int e^{\lambda g} d \mu \leq \frac{C \lambda^{2}}{4} \int|\nabla g|^{2} e^{\lambda g} d \mu \leq \frac{C \lambda^{2}}{4} \int e^{\lambda g} d \mu .
$$

In other words,

and so

$$
\lambda Z^{\prime}(\lambda)-Z(\lambda) \log Z(\lambda) \leq \frac{C \lambda^{2}}{4} Z(\lambda)
$$

$$
\frac{d}{d \lambda} \frac{\log (Z(\lambda))}{\lambda} \leq \frac{C}{4}, \quad \forall \lambda>0
$$

Observe that $\log (Z(\lambda)) / \lambda \rightarrow Z^{\prime}(0) / Z(0)=\int g d \mu$ when $\lambda \rightarrow 0$, so integrating the differential inequality above between 0 and $\lambda>0$, we get that

$$
\int e^{\lambda\left(g-\int g d \mu\right)} d \mu \leq e^{C \lambda^{2} / 4}, \quad \forall \lambda>0
$$

Finally, for all $t \geq 0$

$$
\mu\left(g>\int g d \mu+r\right) \leq e^{-\lambda r} \int e^{\lambda\left(g-\int g d \mu\right)} d \mu \leq \exp \left(C \lambda^{2} / 4-\lambda r\right) .
$$

Optimizing over $\lambda$ gives the following deviation bound:

$$
\mu\left(g>\int g d \mu+r\right) \leq e^{-r^{2} / C}, \quad \forall r \geq 0 .
$$

Now considering $r>r_{o}:=\sqrt{C \log (2)}$, one easily concludes from $(17)$, that $m_{g}$ (the median of $g$ under $\mu$ ) satisfies

which together with (17) yields to

$$
m_{g} \leq \int g d \mu+r_{o}
$$

$$
\mu\left(g>m_{g}+r\right) \leq e^{-\left[r-r_{o}\right]_{+}^{2}}, \quad \forall r \geq 0 .
$$

According to Proposition 1.7, this proves that $\mu$ satisfies the concentration inequality with the announced Gaussian profile. To conclude the proof, it remains to invoke the classical tensorization property of the logSobolev inequality: if $\mu$ satisfies $\mathbf{L S I}(C)$, then for all positive integer $n, \mu^{n}$ also satisfies $\mathbf{L S I}(C)$ (see e.g. [77, Corollary 5.7]). Therefore repeating the preceding calculations with $\mu^{n}$ instead of $\mu$ yields to the conclusion that $\mu$ satisfies the dimension-free concentration inequality with the profile $\alpha(r)=e^{-\frac{1}{C}\left[r-r_{o}\right]_{+}^{2}}, r \geq 0$.

\subsubsection{Some remarks on the KLS conjecture}

First let us recall the famous KLS conjecture due to Kannan, Lovasz and Simonovits [69]. Recall the definitions of isotropic log-concave measures from Section 1.3.3.

Conjecture 2.23 (Kannan, Lovasz and Simonovits [69]). There is some universal constant $C$ such that any isotropic log-concave probability measure satisfies $\mathbf{P I}(C)$. 
This conjecture can be reduced to a concentration of measure problem, as shown in the following result by E. Milman [89].

Theorem 2.24 (E. Milman [89]). The KLS conjecture is equivalent to the following statement: there are some $r_{o}>0$ and $\lambda_{o} \in[0,1 / 2)$ such that for any positive integer $d$, any isotropic log-concave probability measure $\mu$ on $\mathbb{R}^{d}$ satisfies

$$
\mu\left(A_{r_{o}}\right) \geq 1-\lambda_{o}, \quad \forall A \subset \mathbb{R}^{d} \text { s.t } \mu(A) \geq 1 / 2 .
$$

Note that this follows immediately from E. Milman's Theorem 2.20.

Alternative proof. This result also follows immediately from our characterization of Poincaré inequality given in Theorem 2.16. Indeed the class of isotropic and log-concave probability measures is stable under product. Therefore assuming a universal concentration inequality for isotropic log-concave probability measures is exactly the same as assuming a universal dimension-free concentration property for this class of measures. According to Theorems 2.15 and 2.16 this is equivalent to a universal Poincaré inequality.

Taking $f(x)=|x|^{2}$ as a test function in the Poincaré inequality, one sees that the KLS conjecture directly implies the following conjecture called variance conjecture in the literature (that we already encountered in Section 1.3.3).

Conjecture 2.25 (Variance conjecture). There exists a constant $c>0$ such that any isotropic log-concave random vector $X$ with values in $\mathbb{R}^{d}$ satisfies

$$
\operatorname{Var}\left(|X|^{2}\right) \leq c d
$$

Klartag [71] showed that the variance conjecture is true for unconditional isotropic random vectors (an alternative proof by Barthe and Cordero-Erausquin [10] and by Klartag [73]). We recall that a random vector $X=\left(X_{1}, \ldots, X_{d}\right)$ is unconditional if

$$
\operatorname{Law}\left(X_{1}, \ldots, X_{d}\right)=\operatorname{Law}\left(\varepsilon_{1} X_{1}, \ldots, \varepsilon_{d} X_{d}\right)
$$

for any choice of $\varepsilon_{i}= \pm 1$. As we already mentioned, Guédon and Milman [65] proved that for any isotropic log-concave random vector $X$,

$$
\operatorname{Var}\left(|X|^{2}\right) \leq c d^{5 / 3}
$$

which is the currently best estimate. Recently Eldan [46] proved the following striking result: if the variance conjecture is true then the KLS conjecture is true up to a $\log d$ factor.

Let us finally mention the following extension of Klartag's result recently obtained by the author and CorderoErausquin [37] whose proof relies on some transport techniques and transport inequalities.

Theorem 2.26 (Cordero-Erausquin and Gozlan [37]). There exists a constant $C>0$ such that for any logconcave random vector $X$ with values in $\mathbb{R}^{d}$, it holds

$$
\operatorname{Var}\left(|\bar{X}|^{2}\right) \leq c \sum_{i=1}^{d} \mathbb{E}\left[X_{i}^{2}\right]^{2}
$$

where $\bar{X}$ is defined by $\bar{X}_{i}=X_{i}-\mathbb{E}\left[X_{i} \mid X_{1}, \ldots, X_{i-1}\right]$.

Acknowledgements: The author would like to thank the organizers of the "Journées du groupe Modélisation Aléatoire et Statistique de la SMAI" for their invitation to give a plenary talk. He also wants to thank the three anonymous referees for their careful reading and for the many suggestions that greatly improved the presentation of this survey. 


\section{REFERENCES}

1. Shigeki Aida and Daniel W. Stroock, Moment estimates derived from Poincaré and logarithmic Sobolev inequalities, Math. Res. Lett. 1 (1994), no. 1, 75-86. MR 1258492 (95f:60086)

2. Greg W. Anderson, Alice Guionnet, and Ofer Zeitouni, An introduction to random matrices, Cambridge Studies in Advanced Mathematics, vol. 118, Cambridge University Press, Cambridge, 2010. MR 2760897 (2011m:60016)

3. Cécile Ané, Sébastien Blachère, Djalil Chafaï, Pierre Fougères, Ivan Gentil, Florent Malrieu, Cyril Roberto, and Grégory Scheffer, Sur les inégalités de Sobolev logarithmiques, Panoramas et Synthèses [Panoramas and Syntheses], vol. 10, Société Mathématique de France, Paris, 2000, With a preface by Dominique Bakry and Michel Ledoux. MR 1845806 (2002g:46132)

4. Milla Anttila, Keith Ball, and Irini Perissinaki, The central limit problem for convex bodies, Trans. Amer. Math. Soc. 355 (2003), no. 12, 4723-4735. MR 1997580 (2005b:52010)

5. Dominique Bakry and Michel Émery, Diffusions hypercontractives, Séminaire de probabilités, XIX, 1983/84, Lecture Notes in Math., vol. 1123, Springer, Berlin, 1985, pp. 177-206. MR 889476 (88j:60131)

6. Dominique Bakry, Ivan Gentil, and Michel Ledoux, Analysis and geometry of Markov diffusion operators, Grundlehren der Mathematischen Wissenschaften [Fundamental Principles of Mathematical Sciences], vol. 348, Springer, Cham, 2014. MR 3155209

7. Dominique Bakry and Michel Ledoux, Lévy-Gromov's isoperimetric inequality for an infinite-dimensional diffusion generator, Invent. Math. 123 (1996), no. 2, 259-281. MR 1374200 (97c:58162)

8. Franck Barthe, Transportation techniques and Gaussian inequalities, Optimal transportation, geometry and functional inequalities, CRM Series, vol. 11, Ed. Norm., Pisa, 2010, pp. 1-44. MR 2649000 (2011k:49096)

9. Franck Barthe, Patrick Cattiaux, and Cyril Roberto, Interpolated inequalities between exponential and Gaussian, Orlicz hypercontractivity and isoperimetry, Rev. Mat. Iberoam. 22 (2006), no. 3, 993-1067. MR 2320410 (2008k:47097)

10. Franck Barthe and Dario Cordero-Erausquin, Invariances in variance estimates, Proc. Lond. Math. Soc. (3) 106 (2013), no. 1, 33-64. MR 3020738

11. Franck Barthe and Alexander V. Kolesnikov, Mass transport and variants of the logarithmic Sobolev inequality, J. Geom. Anal. 18 (2008), no. 4, 921-979. MR 2438906 (2009i:58048)

12. Franck Barthe, Yutao Ma, and Zhengliang Zhang, Logarithmic Sobolev inequalities for harmonic measures on spheres, J. Math. Pures Appl. (9) 102 (2014), no. 1, 234-248. MR 3212255

13. Franck Barthe and Bernard Maurey, Some remarks on isoperimetry of Gaussian type, Ann. Inst. H. Poincaré Probab. Statist. 36 (2000), no. 4, 419-434. MR 1785389 (2001k:60055)

14. Gérard Ben Arous and Alice Guionnet, Large deviations for Wigner's law and Voiculescu's non-commutative entropy, Probab. Theory Related Fields 108 (1997), no. 4, 517-542. MR 1465640 (98i:15026)

15. Philippe Biane and Dan-Virgil Voiculescu, A free probability analogue of the Wasserstein metric on the trace-state space, Geom. Funct. Anal. 11 (2001), no. 6, 1125-1138. MR 1878316 (2003d:46087)

16. Sergey G. Bobkov, An isoperimetric inequality on the discrete cube, and an elementary proof of the isoperimetric inequality in Gauss space, Ann. Probab. 25 (1997), no. 1, 206-214. MR 1428506 (98g:60033)

17. ___ Isoperimetric and analytic inequalities for log-concave probability measures, Ann. Probab. 27 (1999), no. 4, 1903-1921. MR 1742893 (2001h:60026)

18. __ On concentration of distributions of random weighted sums, Ann. Probab. 31 (2003), no. 1, 195-215. MR 1959791 (2004a:60049)

19. On a theorem of V. N. Sudakov on typical distributions, Zap. Nauchn. Sem. S.-Peterburg. Otdel. Mat. Inst. Steklov. (POMI) 368 (2009), no. Veroyatnost i Statistika. 15, 59-74, 283. MR 2749183 (2012a:60026)

20. Sergey G. Bobkov, Ivan Gentil, and Michel Ledoux, Hypercontractivity of Hamilton-Jacobi equations, J. Math. Pures Appl. (9) 80 (2001), no. 7, 669-696. MR 1846020 (2003b:47073)

21. Sergey G. Bobkov and Friedrich Götze, Exponential integrability and transportation cost related to logarithmic Sobolev inequalities, J. Funct. Anal. 163 (1999), no. 1, 1-28. MR 1682772 (2000b:46059)

22. Sergey G. Bobkov and Christian Houdré, Some connections between isoperimetric and Sobolev-type inequalities, Mem. Amer. Math. Soc. 129 (1997), no. 616, viii+111. MR 1396954 (98b:46038)

23. __ Weak dimension-free concentration of measure, Bernoulli 6 (2000), no. 4, 621-632. MR 1777687 (2001i:28005)

24. Sergey G. Bobkov and Michel Ledoux, Poincaré's inequalities and Talagrand's concentration phenomenon for the exponential distribution, Probab. Theory Related Fields 107 (1997), no. 3, 383-400. MR 1440138 (98e:60026)

25. __ From Brunn-Minkowski to Brascamp-Lieb and to logarithmic Sobolev inequalities, Geom. Funct. Anal. 10 (2000), no. 5, 1028-1052. MR 1800062 (2002k:26028)

26. Vladimir I. Bogachev, Gaussian measures, Mathematical Surveys and Monographs, vol. 62, American Mathematical Society, Providence, RI, 1998. MR 1642391 (2000a:60004)

27. Vladimir I. Bogachev and Alexander V. Kolesnikov, The Monge-Kantorovich problem: achievements, connections, and prospects, Uspekhi Mat. Nauk 67 (2012), no. 5(407), 3-110. MR 3058744

28. François Bolley and Cédric Villani, Weighted Csiszár-Kullback-Pinsker inequalities and applications to transportation inequalities, Ann. Fac. Sci. Toulouse Math. (6) 14 (2005), no. 3, 331-352. MR 2172583 (2006j:60018) 
29. Christer Borell, The Brunn-Minkowski inequality in Gauss space, Invent. Math. 30 (1975), no. 2, 207-216. MR 0399402 (53 \#3246)

30. Aleksandr A. Borovkov and Sergei A. Utev, An inequality and a characterization of the normal distribution connected with it, Teor. Veroyatnost. i Primenen. 28 (1983), no. 2, 209-218. MR 700206 (84g:60029)

31. Eric A. Carlen and James Kerce, On the cases of equality in Bobkov's inequality and Gaussian rearrangement, Calc. Var. Partial Differential Equations 13 (2001), no. 1, 1-18. MR 1854254 (2002f:26016)

32. Patrick Cattiaux and Arnaud Guillin, On quadratic transportation cost inequalities, J. Math. Pures Appl. (9) 86 (2006), no. 4, 341-361. MR 2257848 (2007g:47066)

33. Patrick Cattiaux, Arnaud Guillin, and Li-Ming Wu, A note on Talagrand's transportation inequality and logarithmic Sobolev inequality, Probab. Theory Related Fields 148 (2010), no. 1-2, 285-304. MR 2653230 (2011j:60059)

34. Sourav Chatterjee, Stein's method for concentration inequalities, Probab. Theory Related Fields 138 (2007), no. 1-2, 305-321. MR 2288072 (2008e:60038)

35. Andrea Cianchi, Nicola Fusco, Francesco Maggi, and Aldo Pratelli, On the isoperimetric deficit in Gauss space, Amer. J. Math. 133 (2011), no. 1, 131-186. MR 2752937 (2012b:28007)

36. Dario Cordero-Erausquin, Some applications of mass transport to Gaussian-type inequalities, Arch. Ration. Mech. Anal. 161 (2002), no. 3, 257-269. MR MR1894593 (2003h:49076)

37. Dario Cordero-Erausquin and Nathael Gozlan, Transport proofs of weighted poincar Ál inequalities for log concave distributions, To appear in Bernoulli, 2015.

38. Imre Csiszár, Information-type measures of difference of probability distributions and indirect observations, Studia Sci. Math. Hungar. 2 (1967), 299-318. MR 0219345 (36 \#2428)

39. Amir Dembo, Information inequalities and concentration of measure, Ann. Probab. 25 (1997), no. 2, 927-939. MR 1434131 (98e:60027)

40. Amir Dembo and Ofer Zeitouni, Large deviations techniques and applications, Stochastic Modelling and Applied Probability, vol. 38, Springer-Verlag, Berlin, 2010, Corrected reprint of the second (1998) edition. MR 2571413 (2011b:60094)

41. Persi Diaconis and David Freedman, Asymptotics of graphical projection pursuit, Ann. Statist. 12 (1984), no. 3, 793-815. MR 751274 (86b:62011)

42. Hacène Djellout, Arnaud Guillin, and Li-Ming Wu, Transportation cost-information inequalities and applications to random dynamical systems and diffusions, Ann. Probab. 32 (2004), no. 3B, 2702-2732. MR 2078555 (2005i:60031)

43. Aryeh Dvoretzky, Some results on convex bodies and Banach spaces, Proc. Internat. Sympos. Linear Spaces (Jerusalem, 1960), Jerusalem Academic Press, Jerusalem; Pergamon, Oxford, 1961, pp. 123-160. MR 0139079 (25 \#2518)

44. Antoine Ehrhard, Symétrisation dans l'espace de Gauss, Math. Scand. 53 (1983), no. 2, 281-301. MR 745081 (85f:60058)

45. __ Inégalités isopérimétriques et intégrales de Dirichlet gaussiennes, Ann. Sci. École Norm. Sup. (4) 17 (1984), no. 2, 317-332. MR 760680 (86b:49064)

46. Ronen Eldan, Thin shell implies spectral gap up to polylog via a stochastic localization scheme, Geom. Funct. Anal. 23 (2013), no. 2, 532-569. MR 3053755

47. _ A two-sided estimate for the Gaussian noise stability deficit, Invent. Math. 201 (2015), no. 2, 561-624. MR 3370621

48. Denis Feyel and Ali S. Üstünel, Monge-Kantorovitch measure transportation and Monge-Ampère equation on Wiener space, Probab. Theory Related Fields 128 (2004), no. 3, 347-385. MR 2036490 (2004m:60121)

49. Bruno Fleury, Olivier Guédon, and Grigoris Paouris, A stability result for mean width of $L_{p}$-centroid bodies, Adv. Math. 214 (2007), no. 2, 865-877. MR 2349721 (2008g:52012)

50. Ivan Gentil, Inégalités de Sobolev logarithmiques et hypercontractivité en mécanique statistique et en E.D.P., Thèse de doctorat, Univ. Paul Sabatier Toulouse., 2001.

51. Ivan Gentil, Arnaud Guillin, and Laurent Miclo, Modified logarithmic Sobolev inequalities and transportation inequalities, Probab. Theory Related Fields 133 (2005), no. 3, 409-436. MR 2198019 (2007e:60013)

52. Nathael Gozlan, Integral criteria for transportation-cost inequalities, Electron. Comm. Probab. 11 (2006), 64-77. MR 2231734 (2007h:60016)

53. A characterization of dimension free concentration in terms of transportation inequalities, Ann. Probab. 37 (2009), no. 6, 2480-2498. MR 2573565 (2011a:60079)

54. Poincaré inequalities and dimension free concentration of measure, Ann. Inst. Henri Poincaré Probab. Stat. 46 (2010), no. 3, 708-739. MR 2682264 (2011h:60039)

55. - Transport-entropy inequalities on the line, Electron. J. Probab. 17 (2012), no. 49, 18. MR 2946156

56. Nathael Gozlan and Christian Léonard, Transport inequalities. A survey, Markov Process. Related Fields 16 (2010), no. 4, 635-736. MR 2895086

57. Nathael Gozlan, Cyril Roberto, and Paul-Marie Samson, From concentration to logarithmic Sobolev and Poincaré inequalities, J. Funct. Anal. 260 (2011), no. 5, 1491-1522. MR 2749436 (2012a:60048)

58. _ A new characterization of Talagrand's transport-entropy inequalities and applications, Ann. Probab. 39 (2011), no. 3, 857-880. MR 2789577 (2012d:60049)

59. __ Characterization of Talagrand's transport-entropy inequalities in metric spaces, Ann. Probab. 41 (2013), no. 5, 3112-3139. MR 3127876 
60. Hamilton Jacobi equations on metric spaces and transport entropy inequalities, Rev. Mat. Iberoam. 30 (2014), no. 1, 133-163. MR 3186934

61. __ From dimension free concentration to the Poincaré inequality, Calc. Var. Partial Differential Equations 52 (2015), no. 3-4, 899-925. MR 3311918

62. Mikhael Gromov, Metric structures for Riemannian and non-Riemannian spaces, Progress in Mathematics, vol. 152, Birkhäuser Boston, Inc., Boston, MA, 1999, Based on the 1981 French original [ MR0682063 (85e:53051)], With appendices by M. Katz, P. Pansu and S. Semmes, Translated from the French by Sean Michael Bates. MR 1699320 (2000d:53065)

63. Mikhael Gromov and Vitali D. Milman, A topological application of the isoperimetric inequality, Amer. J. Math. 105 (1983), no. 4, 843-854. MR 708367 (84k:28012)

64. Olivier Guédon, Concentration phenomena in high dimensional geometry, Journées MAS 2012, ESAIM Proc., vol. 44, EDP Sci., Les Ulis, 2014, pp. 47-60. MR 3178607

65. Olivier Guédon and Emanuel Milman, Interpolating thin-shell and sharp large-deviation estimates for isotropic log-concave measures, Geom. Funct. Anal. 21 (2011), no. 5, 1043-1068. MR 2846382

66. Alice Guionnet and Ofer Zeitouni, Concentration of the spectral measure for large matrices, Electron. Comm. Probab. $\mathbf{5}$ (2000), 119-136. MR 1781846 (2001k:15035)

67. Fumio Hiai, Dénes Petz, and Yoshimichi Ueda, Free transportation cost inequalities via random matrix approximation, Probab. Theory Related Fields 130 (2004), no. 2, 199-221. MR 2093762 (2005k:46177)

68. Aldéric Joulin and Yann Ollivier, Curvature, concentration and error estimates for Markov chain Monte Carlo, Ann. Probab. 38 (2010), no. 6, 2418-2442. MR 2683634 (2011j:60229)

69. Ravi Kannan, László Lovász, and Miklós Simonovits, Isoperimetric problems for convex bodies and a localization lemma, Discrete Comput. Geom. 13 (1995), no. 3-4, 541-559. MR 1318794 (96e:52018)

70. Bo'az Klartag, A central limit theorem for convex sets, Invent. Math. 168 (2007), no. 1, 91-131. MR 2285748 (2008d:60034)

71. __ A Berry-Esseen type inequality for convex bodies with an unconditional basis, Probab. Theory Related Fields $\mathbf{1 4 5}$ (2009), no. 1-2, 1-33. MR 2520120 (2010h:60072)

72. __ High-dimensional distributions with convexity properties, European Congress of Mathematics, Eur. Math. Soc., Zürich, 2010, pp. 401-417. MR 2648334 (2011d:46028)

73. __ Poincaré inequalities and moment maps, Ann. Fac. Sci. Toulouse Math. (6) 22 (2013), no. 1, 1-41. MR 3247770

74. Solomon Kullback, A lower bound for discrimination information in terms of variation, IEEE Trans. Inform. Theory 4 (1967), $126-127$.

75. Rafał Latała and Krzysztof Oleszkiewicz, Between Sobolev and Poincaré, Geometric aspects of functional analysis, Lecture Notes in Math., vol. 1745, Springer, Berlin, 2000, pp. 147-168. MR 1796718 (2002b:60025)

76. Michel Ledoux, On Talagrand's deviation inequalities for product measures, ESAIM Probab. Statist. 1 (1995/97), 63-87 (electronic). MR 1399224 (97j:60005)

77. The concentration of measure phenomenon, Mathematical Surveys and Monographs, vol. 89, American Mathematical Society, Providence, RI, 2001. MR 1849347 (2003k:28019)

78. Michel Ledoux and Ionel Popescu, Mass transportation proofs of free functional inequalities, and free Poincaré inequalities, J. Funct. Anal. 257 (2009), no. 4, 1175-1221. MR 2535467 (2011e:46106)

79. Mylène Maïda and Édouard Maurel-Segala, Free transport-entropy inequalities for non-convex potentials and application to concentration for random matrices, Probab. Theory Related Fields 159 (2014), no. 1-2, 329-356. MR 3201924

80. Katalin Marton, A simple proof of the blowing-up lemma, IEEE Trans. Inform. Theory 32 (1986), no. 3, 445-446. MR 838213 (87e:94018)

81. __ Bounding $\bar{d}$-distance by informational divergence: a method to prove measure concentration, Ann. Probab. 24 (1996), no. 2, 857-866. MR 1404531 (97f:60064)

82. _ A measure concentration inequality for contracting Markov chains, Geom. Funct. Anal. 6 (1996), no. 3, 556-571. MR 1392329 (97g:60082)

83. Pascal Massart, About the constants in Talagrand's concentration inequalities for empirical processes, Ann. Probab. 28 (2000), no. 2, 863-884. MR 1782276 (2001m:60038)

84. __ Concentration inequalities and model selection, Lecture Notes in Mathematics, vol. 1896, Springer, Berlin, 2007, Lectures from the 33rd Summer School on Probability Theory held in Saint-Flour, July 6-23, 2003, With a foreword by Jean Picard. MR 2319879 (2010a:62008)

85. Bernard Maurey, Some deviation inequalities, Geom. Funct. Anal. 1 (1991), no. 2, 188-197. MR 1097258 (92g:60024)

86. Colin McDiarmid, On the method of bounded differences, Surveys in combinatorics, 1989 (Norwich, 1989 ), London Math. Soc. Lecture Note Ser., vol. 141, Cambridge Univ. Press, Cambridge, 1989, pp. 148-188. MR 1036755 (91e:05077)

87. _ Concentration, Probabilistic methods for algorithmic discrete mathematics, Algorithms Combin., vol. 16, Springer, Berlin, 1998, pp. 195-248. MR 1678578 (2000d:60032)

88. Emanuel Milman, On the role of convexity in isoperimetry, spectral gap and concentration, Invent. Math. 177 (2009), no. 1, 1-43. MR 2507637 (2010j:28004)

89. __ Isoperimetric and concentration inequalities: equivalence under curvature lower bound, Duke Math. J. 154 (2010), no. 2, 207-239. MR 2682183 (2011g:53079) 
90. Vitali D. Milman, A new proof of A. Dvoretzky's theorem on cross-sections of convex bodies, Funkcional. Anal. i Priložen. 5 (1971), no. 4, 28-37. MR 0293374 (45 \#2451)

91. Vitali D. Milman and Gideon Schechtman, Asymptotic theory of finite-dimensional normed spaces, Lecture Notes in Mathematics, vol. 1200, Springer-Verlag, Berlin, 1986, With an appendix by M. Gromov. MR 856576 (87m:46038)

92. Elchanan Mossel and Joe Neeman, Robust dimension free isoperimetry in Gaussian space, Ann. Probab. 43 (2015), no. 3, 971-991. MR 3342656

93. Felix Otto and Cédric Villani, Generalization of an inequality by Talagrand and links with the logarithmic Sobolev inequality, J. Funct. Anal. 173 (2000), no. 2, 361-400. MR 1760620

94. M. S. Pinsker, Information and information stability of random variables and processes, Translated and edited by Amiel Feinstein, Holden-Day, Inc., San Francisco, Calif.-London-Amsterdam, 1964. MR 0213190 (35 \#4054b)

95. Gilles Pisier, The volume of convex bodies and Banach space geometry, Cambridge Tracts in Mathematics, vol. 94, Cambridge University Press, Cambridge, 1989. MR 1036275 (91d:52005)

96. Svetlozar T. Rachev and Ludger Rüschendorf, Mass transportation problems. Vol. I, Probability and its Applications (New York), Springer-Verlag, New York, 1998, Theory. MR 1619170 (99k:28006)

97. Sebastian Riedel, Talagrand's transportation-cost inequality and applications to (rough) path spaces., Preprint arXiv:1403.2585, 2013.

98. Paul-Marie Samson, Concentration of measure inequalities for Markov chains and $\Phi$-mixing processes, Ann. Probab. 28 (2000), no. 1, 416-461. MR 1756011 (2001d:60015)

99. Concentration inequalities for convex functions on product spaces, Stochastic inequalities and applications, Progr. Probab., vol. 56, Birkhäuser, Basel, 2003, pp. 33-52. MR 2073425 (2005d:60035)

100. _ Infimum-convolution description of concentration properties of product probability measures, with applications, Ann. Inst. H. Poincaré Probab. Statist. 43 (2007), no. 3, 321-338. MR 2319700 (2008c:60010)

101. Michael Schmuckenschläger, Martingales, Poincaré type inequalities, and deviation inequalities, J. Funct. Anal. 155 (1998), no. 2, 303-323. MR 1624545 (99h:60037)

102. Vladimir N. Sudakov, Typical distributions of linear functionals in finite-dimensional spaces of high dimension, Dokl. Akad. Nauk SSSR 243 (1978), no. 6, 1402-1405. MR 517198 (80b:60011)

103. Vladimir N. Sudakov and Boris S. Cirel'son, Extremal properties of half-spaces for spherically invariant measures, Zap. Naučn. Sem. Leningrad. Otdel. Mat. Inst. Steklov. (LOMI) 41 (1974), 14-24, 165, Problems in the theory of probability distributions, II. MR 0365680 (51 \#1932)

104. Michel Talagrand, A new isoperimetric inequality and the concentration of measure phenomenon, Geometric aspects of functional analysis (1989-90), Lecture Notes in Math., vol. 1469, Springer, Berlin, 1991, pp. 94-124. MR 1122615 (93d:60095)

105. _ Concentration of measure and isoperimetric inequalities in product spaces, Inst. Hautes Études Sci. Publ. Math. (1995), no. 81, 73-205. MR 1361756 (97h:60016)

106. Transportation cost for Gaussian and other product measures, Geom. Funct. Anal. 6 (1996), no. 3, 587-600. MR 1392331 (97d:60029)

107. Cédric Villani, Topics in optimal transportation, Graduate Studies in Mathematics, vol. 58, American Mathematical Society, Providence, RI, 2003. MR 1964483

108. _ Optimal transport, Grundlehren der Mathematischen Wissenschaften [Fundamental Principles of Mathematical Sciences], vol. 338, Springer-Verlag, Berlin, 2009, Old and new. MR 2459454 (2010f:49001)

109. Heinrich von Weizsäcker, Sudakov's typical marginals, random linear functionals and a conditional central limit theorem, Probab. Theory Related Fields 107 (1997), no. 3, 313-324. MR 1440135 (99c:60006)

110. Feng-Yu Wang, Logarithmic Sobolev inequalities on noncompact Riemannian manifolds, Probab. Theory Related Fields 109 (1997), no. 3, 417-424. MR 1481127 (98i:58253) 